\title{
Tanshinone IIA attenuates cerebral aneurysm formation by inhibiting the NF-кB-mediated inflammatory response
}

\author{
JUN MA ${ }^{1}$, DAORONG HOU ${ }^{2}$, ZHIQING WEI ${ }^{3}$, JIANGUO ZHU ${ }^{4}, \mathrm{HUA} \mathrm{LU}^{5}$, ZHENG LI $^{5}$, \\ XIEFENG WANG ${ }^{5}$, YINGBIN LI ${ }^{1}$, GUANQUN QIAO ${ }^{1}$ and NING LIU $^{5}$ \\ ${ }^{1}$ Department of Neurosurgery, The Second Affiliated Hospital of Nanjing Medical University; \\ ${ }^{2}$ Key Laboratory of The Model Animal Research, Animal Core Facility of Nanjing Medical University, \\ Nanjing Medical University; Departments of ${ }^{3}$ Vascular Surgery and ${ }^{4}$ Radiology, The Second Affiliated Hospital of \\ Nanjing Medical University; ${ }^{5}$ Department of Neurosurgery, The First Affiliated Hospital of Nanjing Medical University, \\ Nanjing, Jiangsu 210029, P.R. China
}

Received November 7, 2018; Accepted May 24, 2019

DOI: $10.3892 / \mathrm{mmr} .2019 .10407$

\begin{abstract}
The inflammatory response plays a vital role in cerebral aneurysm (CA) formation and progression. Tanshinone IIA (Tan IIA) is one of the major active components of Chinese medicine Danshen (Salvia miltiorrhiza Bunge) and is widely used for the treatment of cardiovascular diseases, due to its anti-inflammatory effects. The aim of the present study was to investigate whether Tan IIA can attenuate CA formation in rat models, and determine its underlying mechanisms. CAs were induced in rats surgically and through high-salt diet treatments. The Tan IIA-treated group displayed relatively mild symptoms, as compared with the control group. Tan IIA treatment reduced macrophage infiltration and nuclear factor (NF)- $\kappa \mathrm{B}$ activation in aneurysmal walls. Next, lipopolysaccharide (LPS)-stimulated RAW 264.7 murine macrophage cells were used to examine the anti-inflammatory effects of Tan IIA on macrophages. It was found that Tan IIA reversed LPS-induced differentiation of RAW 264.7 cells and suppressed NF- $\kappa \mathrm{B}$ pathway activation. In conclusion, these findings demonstrated that Tan IIA can suppress CA formation by inhibiting inflammatory responses in macrophages.
\end{abstract}

\section{Introduction}

Cerebral aneurysm (CA) is one of the most common clinical cerebrovascular diseases, occurring in 1-5\% of the general population (1). Most unruptured CAs are thought to be asymptomatic or present with common symptoms, such as chronic headache (2). Anatomical features, demographic

Correspondence to: Dr Ning Liu, Department of Neurosurgery, The First Affiliated Hospital of Nanjing Medical University, 300 Guangzhou Road, Nanjing, Jiangsu 210029, P.R. China E-mail: liuning0801@126.com

Key words: tanshinone IIA, cerebral aneurysm, nuclear factor- $\kappa \mathrm{B}$, inflammation characteristics and medical conditions were confirmed to be associated with the increasing risk of rupture in CAs. Ruptured CAs are the most common cause of subarachnoid hemorrhage (SAH). SAH affects 6-12 individual per 100,000 of the population each year and causes worldwide mortality and morbidity. Approximately 50\% of SAH patients succumb to the disease, and half of the surviving patients suffer from complications. Although microsurgical clipping and endovascular coiling have become established therapies for CAs, effective noninvasive medical therapies are still indispensable $(3,4)$. Consequently, it is crucial to discover novel effective medical treatments to prevent CA growth and rupture.

Numerous studies have suggested that chronic inflammation plays an important role in CA formation and progression (5-9). Nuclear factor (NF)- $\kappa B$-mediated macrophage recruitment through the upregulation of monocyte chemoattractant protein-1 (MCP-1) expression is an important process in the inflammatory response. NF- $\mathrm{kB}$ also regulates the expression of inflammation-associated genes, such as interleukin (IL)-1 $\beta$, inducible nitric oxide synthase (iNOS) and matrix metalloproteinases (MMPs) (10). IL-1 $\beta$ and iNOS may cause apoptosis in vascular smooth muscle cells, leading to endothelial damage and disruption of the internal elastic lamina (11). MMP-2 and MMP-9 are involved in vessel wall remodeling occurring as a result of collagen degradation and breakdown (12). Due to its vital role in $\mathrm{CA}, \mathrm{NF}-\mathrm{\kappa B}$ may serve as a therapeutic target for CA treatment.

Tanshinone IIA (Tan IIA) is a major component isolated from a traditional Chinese medicine called Danshen (Salvia miltiorrhiza Bunge), which is widely used for the treatment of various diseases, such as cardiovascular diseases, diabetes and cancer (13-15). Tan IIA was found to inhibit the tumor necrosis factor (TNF)- $\alpha$-induced increase in phospho-AKT and NF- $\mathrm{BB}$ DNA-binding, leading to human aortic smooth muscle cell migration and increased MMP-9 activity (16). However, the function of Tan IIA in CA treatment has not yet been reported.

The main aim of the present study was to investigate the role and possible mechanism of Tan IIA in CA formation in rat models, and to provide potential clinical therapeutic 
approaches for the suppression of CA formation and subarachnoid hemorrhage.

\section{Materials and methods}

Induction of CAs in rats. All animal experiments were performed in accordance with the Guide for the Care and Use of Laboratory Animals by the National Institutes of Health. The handling procedures were approved by the Institutional Review Board of Nanjing Medical University. Male Sprague Dawley rats ( $n=6 /$ group) aged 7 weeks were used for CA induction, as previously described (17). All rats were housed on hard wood chip bedding at $23^{\circ} \mathrm{C}$ and supplied with standard rat chow and water ad libitum. The housing room followed a 12-h light/dark cycle (lights on at 19:00 h). The rats underwent surgery under general anesthesia with ketamine/xylazine [ketamine $80 \mathrm{mg} / \mathrm{kg}$, xylazine $10 \mathrm{mg} / \mathrm{mg}$ intraperitoneal (i.p.) injection], and the left common carotid artery, which induced hemodynamic stress, and left renal artery were ligated simultaneously. The treated rats were then housed in standard facilities fed a high-salt diet containing $8 \%$ sodium chloride to induce systemic hypertension. Blood pressure was measured by the tail-cuff method without anesthesia (Kent Scientific Corp., Torrington, CT, USA). Rats were euthanized by deep anesthesia (ketamine $240 \mathrm{mg} / \mathrm{kg}$, xylazine $30 \mathrm{mg} / \mathrm{kg}$, i.p. injection) 4 weeks after CA induction, and then perfused transcardially with phosphate-buffered saline followed by $4 \%$ paraformaldehyde. The sacrifice of the rats after deep anesthesia was confirmed by observing for the absence of movement, respiratory and heartbeat activity for at least 3 min. Degenerative change assessments of vascular walls were performed using Verhoeff-Van Gieson staining. Aneurysm size and vascular wall thickness were assessed by independent observers in a blinded manner. Macrophage infiltration was examined by hematoxylin and eosin staining.

RNA isolation and reverse-transcription quantitative polymerase chain reaction $(R T-q P C R)$. Total RNA was extracted from homogenized tissues using TRIzol reagent (Thermo Fisher Scientific, Inc., Waltham, MA, USA), according to the manufacturer's instructions. cDNAs were reversed transcribed from RNA samples using PrimeScript 1st Strand cDNA Synthesis kit (Takara Bio, Inc., Otsu, Japan) and applied as templates. RT-qPCR was performed using an ABI 7500 qPCR system (Thermo Fisher Scientific, Inc.) with FastStart Universal SYBR Green Master (Roche Diagnostics, Indianapolis, IN, USA), according to the manufacturer's instructions. The cycling steps of the reactions were $94^{\circ} \mathrm{C}$ for $15 \mathrm{sec}, 60^{\circ} \mathrm{C}$ for $30 \mathrm{sec}$ and $72^{\circ} \mathrm{C}$ for $30 \mathrm{sec}$. Each reaction was performed in triplicate in a final volume of $20 \mu \mathrm{l}$. RT-qPCR data were analyzed following the $2^{-\Delta \Delta \mathrm{Cq}}$ method and normalized to GAPDH (18). Primers used were as followed: NF- $\mathrm{B}$ forward 5'-ACGATCTGTTTCCCCTCATC-3' and reverse 5'-TGC TTCTCTCCCCAGGAATA-3'; MCP-1 forward 5'-CCTCCA CCACTATGCAGGTCTC-3' and reverse 5'-GCACGTGGA TGCTACAGGC-3'; MMP-2 forward 5'-CTGATAACCTGG ATGCAGTCGT-3' and reverse 5'-CCAGCCAGTCCGATT TGA-3'; MMP-9 forward 5'-TTCAAGGACGGTCGGTATT-3' and reverse 5'-CTCGAGCCTAGACCCAACTTA-3'; GAPDH forward 5'-AAGAAGGTGGTGAAGCAGGC-3' and reverse 5'-TCCACCACCCTGTTGCTGTA-3'.

Western blotting assay. Proteins were isolated from homogenized tissues that were incubated with ice-cold RIPA lysis buffer containing $1 \mathrm{mM}$ PMSF for $10 \mathrm{~min}$ and centrifuged at $12,000 \mathrm{x} \mathrm{g}$ for $10 \mathrm{~min}$ at $4^{\circ} \mathrm{C}$. Protein concentration was measured by Pierce BCA Protein Assay kit (Thermo Fisher Scientific, Inc.). Protein (40 mg) was separated using a $10 \%$ SDS-PAGE gel and transferred onto $0.2 \mu \mathrm{m}$ PVDF membranes (Roche Diagnostics). Following blocking with 3\% (w/v) BSA in TBST buffer at room temperature, the membranes were incubated with primary antibodies overnight at $4^{\circ} \mathrm{C}$. HRP-conjugated secondary antibodies were incubated with washed membranes for $1 \mathrm{~h}$ at room temperature. Pierce ECL Western Blotting Substrate (Thermo Fisher Scientific, Inc.) was used to detect the proteins. Antibodies used in this assay

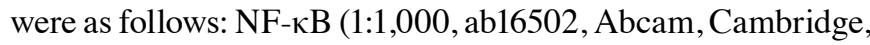
USA), actin (1:5,000, ab179467, Abcam), Lamin B (1:10,000, ab16048), MMP-9 (1:2,000, ab76003, Abcam) and GAPDH (1:5,000, AC002, ABclonal Biotech Co., Ltd., Wuhan, China).

Cell culture. The murine macrophage RAW 264.7 cell line was cultured in 6-well plates at a density of $1 \times 10^{6}$ cells per well. Cells were maintained in DMEM with $10 \%$ (v/v) FBS, $100 \mathrm{IU} / \mathrm{ml}$ penicillin $\mathrm{G}, 100 \mu \mathrm{g} / \mathrm{ml}$ streptomycin, and $2 \mathrm{mM}$ L-glutamine at $37^{\circ} \mathrm{C}$ in a humidified atmosphere containing $5 \% \mathrm{CO}_{2}$. To set up the Tan IIA-treated groups, the cells were treated the next day with or without $10 \mu \mathrm{M}$ Tan IIA (Merck KGaA, Darmstadt, Germany) in DMSO for $2 \mathrm{~h}$, and then stimulated with $1 \mu \mathrm{g} / \mathrm{ml}$ LPS or treated with DMSO (control) for $24 \mathrm{~h}$.

Cell proliferation and migration assays. The viability of RAW 264.7 cells was assessed using the Cell Counting Kit-8 kit (CCK-8, Dojindo, Japan). RAW 264.7 cells were plated at $1 \times 10^{4}$ in 96 -well plates containing 6 parallel wells and treated with DMSO, LPS or LPS+Tan IIA. During the following $72 \mathrm{~h}$ of incubation, $10 \mu \mathrm{l}$ CCK-8 was added into each well every $24 \mathrm{~h}$. The plates were incubated with CCK-8 for $2 \mathrm{~h}$, and then the absorbance value was measured at $450 \mathrm{~nm}$ wavelength every $24 \mathrm{~h}$ using a microplate reader (PerkinElmer, Inc., Waltham, MA, USA).

Next, $1 \times 10^{5}$ RAW 264.7 cells were suspended into the upper chamber of the Transwell apparatus (Corning Inc., Corning, NY, USA) in $100 \mathrm{ml}$ serum-free medium, while the bottom chamber contained medium with $10 \%$ FBS. Following incubation for $24 \mathrm{~h}$, the cells on the upper surface that did not pass through the filter were removed using a moistened cotton swab. The cells on the lower membrane surface were fixed with pure methanol and stained with $0.1 \%$ crystal violet for $20 \mathrm{~min}$. The migrated cells were observed and counted using an inverted microscope at x100 magnification (Olympus Corporation, Tokyo, Japan). Cell migration was expressed as a percentage of DMSO control and results are shown as mean \pm standard deviation from 3 independent experiments.

ELISA. Following the different treatments, supernatants of the RAW 264.7 cells were collected for ELISA. Rat TNF $\alpha$, Rat IL-6 and Rat MCP-1/CCL2 ELISA kits (Wuhan Boster 
A
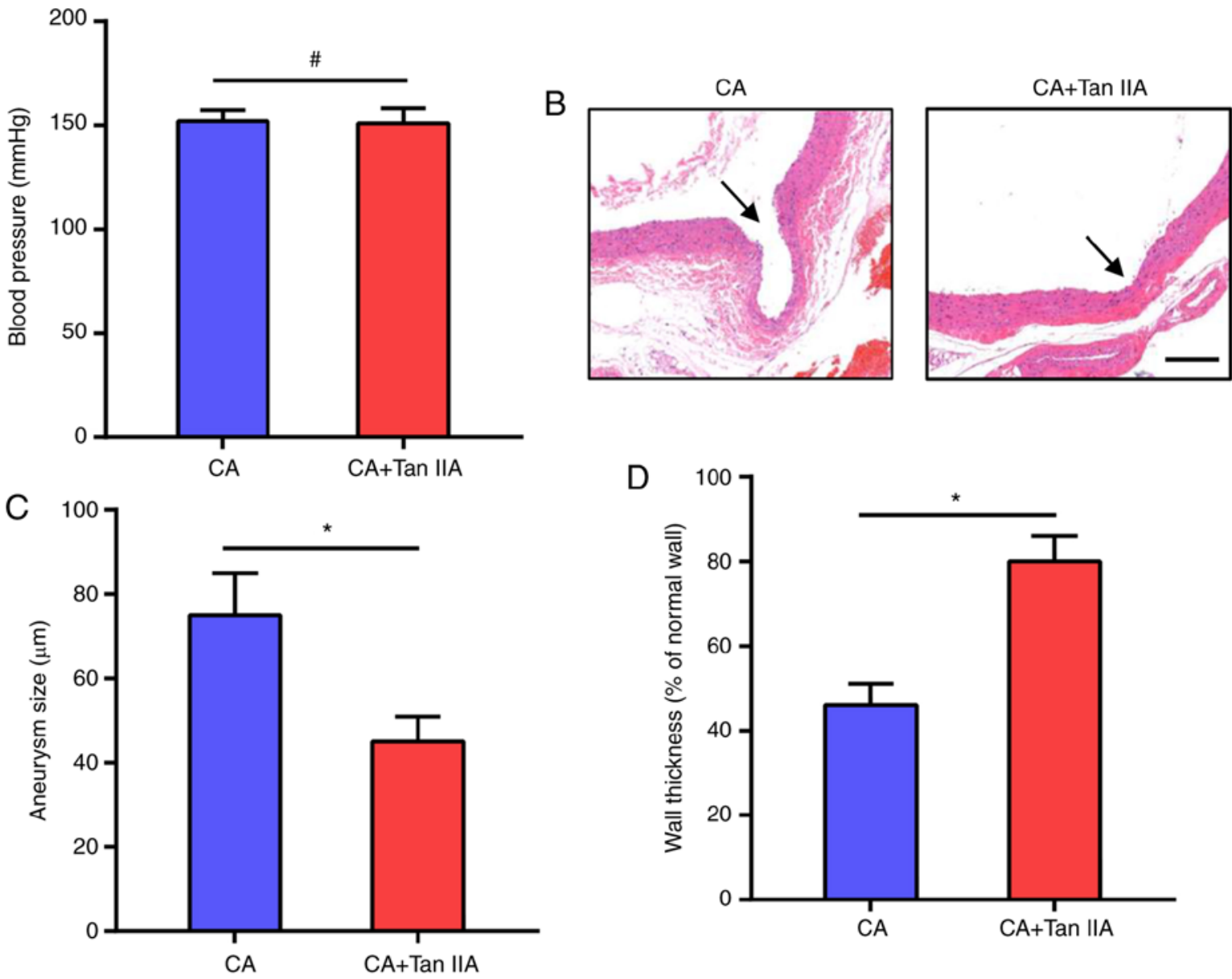

Figure 1. Tan IIA suppresses CA formation in rats. (A) Blood pressure was monitored following CA induction. No significant difference was observed between the $\mathrm{CA}$ and $\mathrm{CA}+\mathrm{Tan}$ IIA group. Data are presented as the mean \pm standard deviation ( $\mathrm{n}=6 /$ group). ${ }^{\text {\#P}} \mathrm{P}>0.05$. (B) Verhoeff-Van Gieson staining of the vascular wall. Arrows indicate the luminal sides of CAs. Scale bar, $30 \mu \mathrm{m}$. (C) Measurement of aneurysm size. (D) Wall thickness ratio. Results are presented as the mean \pm standard deviation ( $\mathrm{n}=6 /$ group). ${ }^{*} \mathrm{P}<0.05$ vs. the CA group. Tan IIA, tanshinone IIA; CA, cerebral aneurysm.

Biological Technology, Ltd., Wuhan, China) and Rat Pro IL1 $\beta$ ELISA kit (Wuhan Abebio Science Co., Ltd., Wuhan, China) were used to measured TNF $\alpha$, IL-6, MCP-1 and IL- $1 \beta$ in the cell supernatants, respectively, according to the manufacturer's instructions.

Gelatin zymography assay. To measure gelatinolytic activities of MMP-9 in the treated cells, gelatin zymography assay was performed as previously described (19). Briefly, protein concentration was measured and protein $(100 \mathrm{mg})$ was mixed with $4 \mathrm{X}$ non-reducing sample buffer at room temperature for 10 min and subjected to $10 \%$ SDS-PAGE containing $0.1 \%$ $(\mathrm{w} / \mathrm{v})$ gelatin. The electrophoresis was performed at a constant voltage $(125 \mathrm{~V})$ for $90 \mathrm{~min}$ at $4^{\circ} \mathrm{C}$. The gel was then incubated with $1 \mathrm{X}$ diluted renaturing solution containing $2.5 \%(\mathrm{v} / \mathrm{v})$ Trixon-100 solution for 30 min twice at room temperature with gentle agitation. The gel was then washed with $1 \mathrm{X}$ developing buffer for an additional $30 \mathrm{~min}$ at room temperature, and the buffer was replaced with fresh developing buffer and incubated at $37^{\circ} \mathrm{C}$ overnight. A staining solution was used to stain the gel for at least $1 \mathrm{~h}$ and the gel was destained with destaining solution until MMP-9 gelatinolytic activity appeared as clearly transparent bands $(92 \mathrm{kDa})$ against the blue background.
Statistical analysis. All results are expressed as the mean \pm standard deviation from multiple independent experiments. The Student's t-test was used to derive the significance between mean values of two groups and one-way ANOVA with Tukey's honestly significant difference (HSD) post hoc test was used in multiple comparisons. Data analysis was performed with the statistical program GraphPad Prism (GraphPad Software, Inc.). P $<0.05$ was considered to indicate a statistically significant difference.

\section{Results}

Tan IIA suppresses $C A$ formation in rats. To investigate the effect of Tan IIA on CA formation, Tan IIA was injected i.p. into rats for 4 weeks after CA formation. The CAs in the rat models were induced by systemic hypertension, thus we first examined the effect of Tan IIA on blood pressure. As shown in Fig. 1A, Tan IIA did not affect blood pressure which was increased in the rats after the surgeries. The morphologic evaluation suggested that Tan IIA treatment suppressed the growth of CAs in the rats (Fig. 1B). The CA+Tan IIA group exhibited a significant decrease in aneurysm size, as compared with the CA group (Fig. 1C). Tan IIA treatment also increased wall thickness, as compared with the CA group (Fig. 1D). 
A
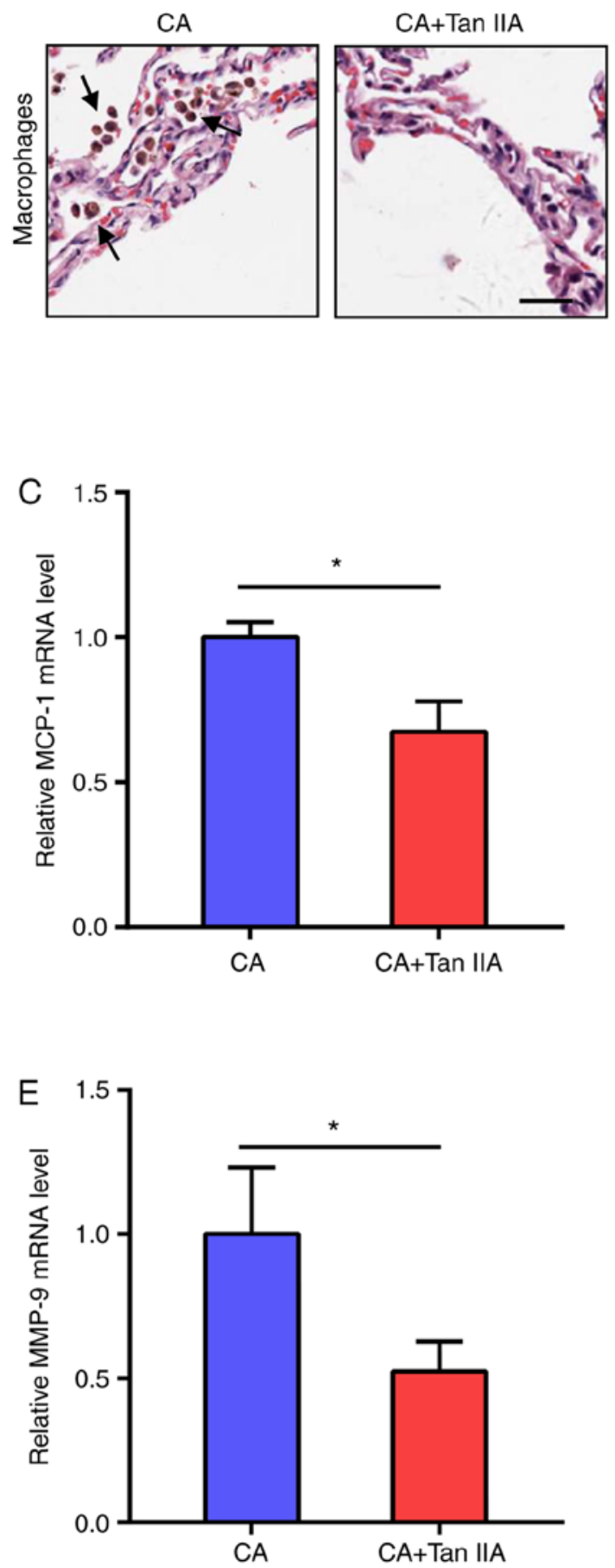

B

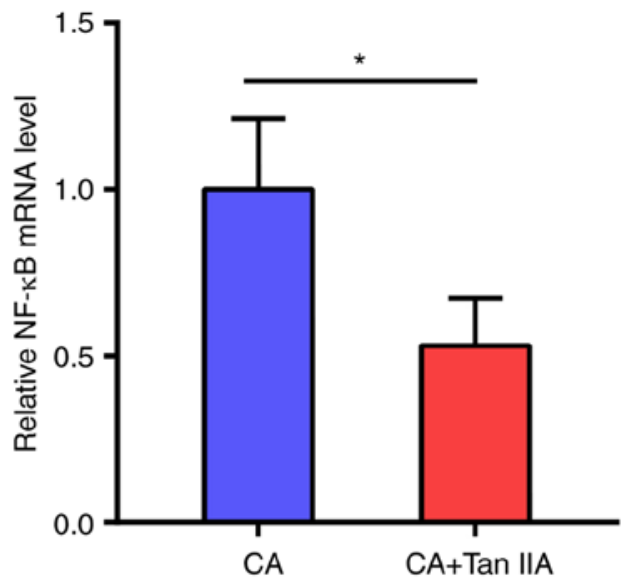

D

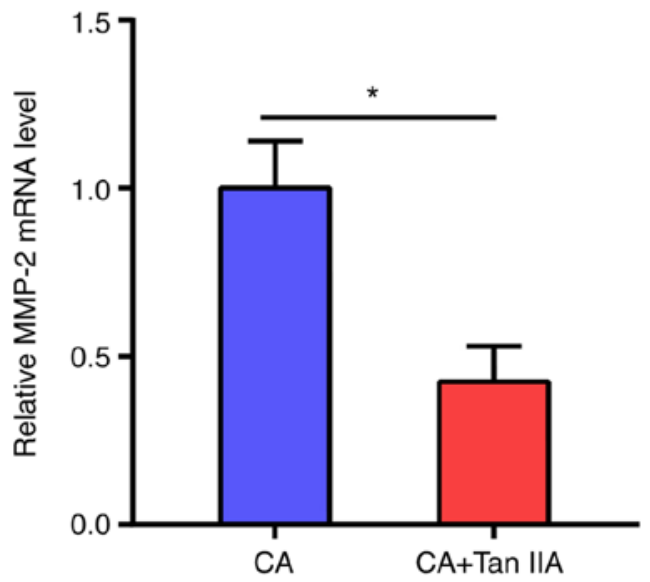

$\mathrm{F}$

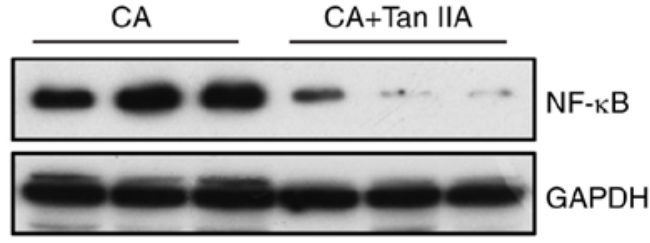

G

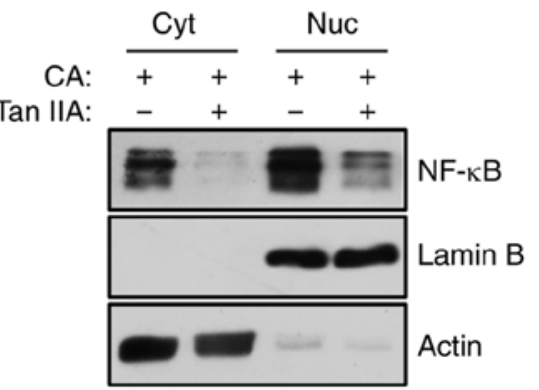

Figure 2. Tan IIA reduces the inflammatory response in aneurysmal walls. (A) Representative images of H\&E staining of aneurysmal walls. Arrows indicate macrophages. Scale bar, $10 \mu \mathrm{m}$. (B-E) RT-qPCR analysis of mRNA expression levels of NF- $\mathrm{B}$ (B) MCP-1 (C) MMP-2 (D) and MMP-9 (E) normalized to GAPDH in the CA and CA+Tan IIA groups. Data are presented as the mean \pm standard deviation ( $\mathrm{n}=6 /$ group), ${ }^{*} \mathrm{P}<0.05$ vs. the $\mathrm{CA}$ group. (F) Western blot analysis of NF- $\mathrm{kB}$ expression levels in the aneurysmal walls of the CA and CA+Tan IIA groups (n=3/group). GAPDH served as the loading control. (G) Western blot analysis of NF-kB distribution in aneurysmal walls of the CA and CA+Tan IIA groups. Cyt, cytoplasm, Nuc, nucleus. Lamin B served as the loading control of the nucleus. Actin served as the loading control of the cytoplasm. Tan IIA, tanshinone IIA; CA, cerebral aneurysm; RT-qPCR, reverse

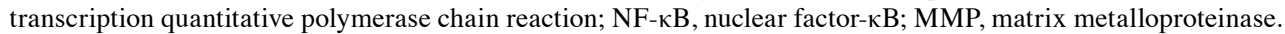

These data suggest that Tan IIA can inhibit CA formation in rat models.

Tan IIA reduces inflammatory response in aneurysmal walls. As macrophage-mediated chronic inflammation plays a vital role in CA formation, the effects of Tan IIA on macrophage infiltration into the CAs were examined. It was found that the $\mathrm{CA}+$ Tan IIA group exhibited less macrophage infiltration, as compared with the CA group (Fig. 2A). As the NF-kB signaling network is essential for macrophage infiltration, NF- $\mathrm{KB}$ and 
A
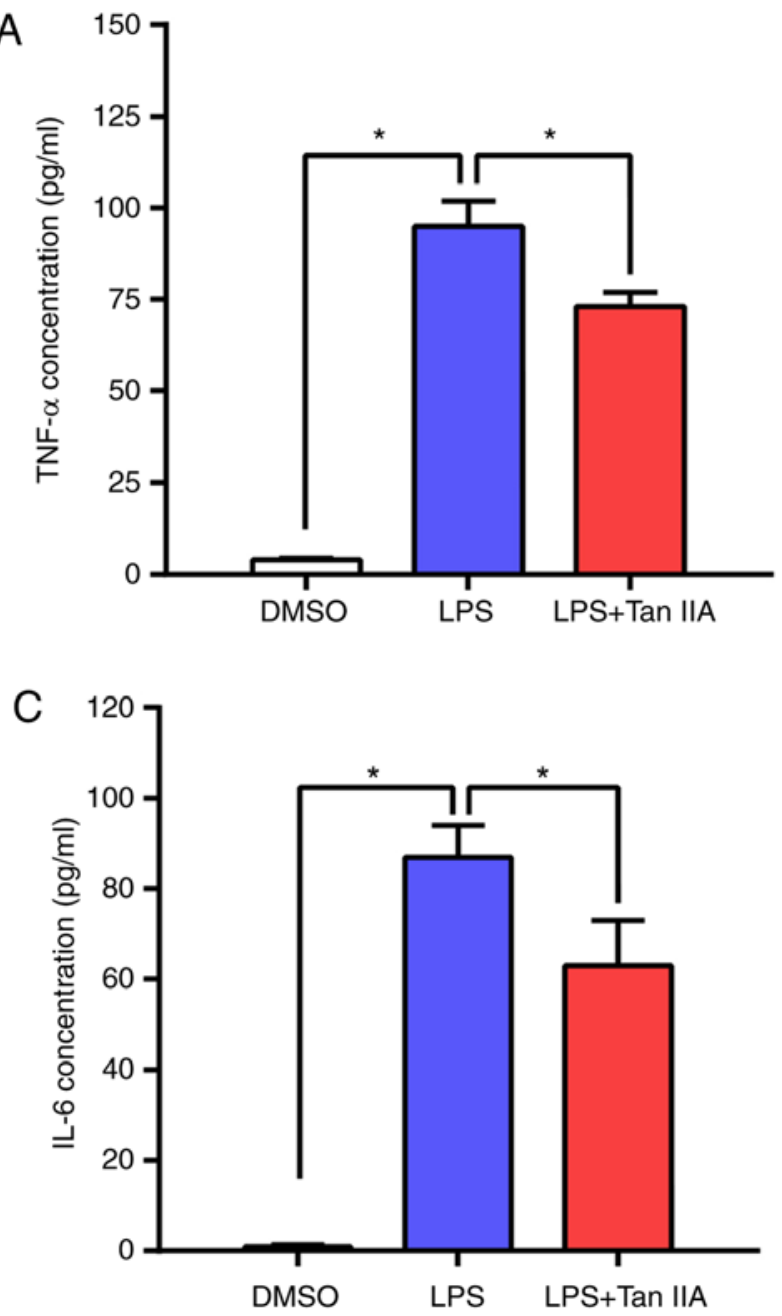

B
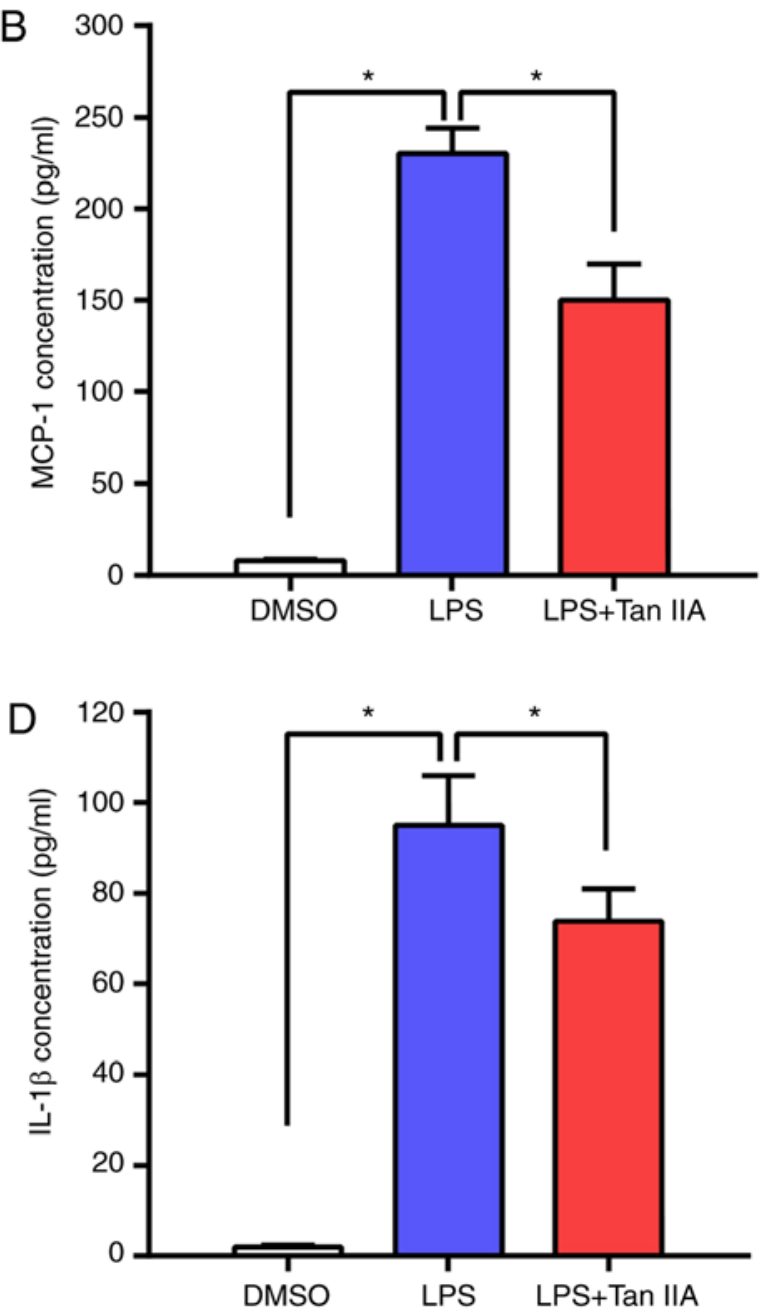

Figure 3. Tan IIA treatment ameliorates inflammatory cytokine upregulation in LPS-stimulated RAW 264.7 cells. The cells were treated with DMSO, LPS $(1 \mu \mathrm{g} / \mathrm{ml})$ or LPS $(1 \mu \mathrm{g} / \mathrm{ml})+$ Tan IIA $(10 \mu \mathrm{M})$ for $24 \mathrm{~h}$. (A-D) ELISA assays were utilized to examine the concentration of four inflammatory cytokines: (A) TNF- $\alpha$, (B) MCP-1, (C) IL-6 and (D) IL-1 $\beta$ in the RAW 264.7 cells. Results are presented as the mean \pm standard deviation. *P<0.05 vs. the indicated control. Tan IIA, tanshinone IIA; LPS, lipopolysaccharide; MCP-1, monocyte chemoattractant protein-1; TNF $\alpha$, tumor necrosis factor $\alpha$; IL, interleukin.

downstream MCP-1, MMP-2 and MMP-9 mRNA levels in the aneurysmal walls were tested. The results indicated that the expression levels of these genes were significantly reduced in the CA+Tan IIA group, as compared with the CA group (Fig. 2B-E). In addition, $N F-\kappa B$ protein levels were decreased in the rats treated with Tan IIA (Fig. 2F and G). These data suggested that the $\mathrm{NF}-\kappa \mathrm{B}$ pathway in macrophages is the target of Tan IIA in the suppression of CA formation.

Tan IIA treatment ameliorates inflammatory cytokine upregulation in LPS-stimulated RAW 264.7 cells. RAW 264.7 is an Abelson murine leukemia virus transformed macrophage-like cell line derived from BALB/c mice and is commonly used as an appropriate model of macrophages (20). LPS-stimulated RAW 264.7 murine macrophage cells were used to evaluate the anti-inflammatory effect of Tan IIA. The concentrations of four main inflammatory cytokines (TNF $\alpha$, MCP-1, IL-6 and IL-1 $\beta$ ) in RAW 264.7 cells were detected by ELISA. As shown in Fig. 3A-D, LPS led to an elevated production of these inflammatory cytokines in RAW 264.7 cells, while Tan IIA treatment attenuated this effect. These results indicated that Tan IIA can ameliorate the inflammatory response triggered by LPS by reducing cytokine production in RAW 264.7 cells.

Tan IIA restores the proliferation and migration capacity of LPS-stimulated RAW 264.7 cells. It has been reported that LPS stimulation induces the differentiation of RAW 264.7 cells and inhibits their proliferation (21). The present results indicated that Tan IIA can reverse this process and increase the proliferation of RAW 264.7 cells (Fig. 4A). Next, the effect of Tan IIA on LPS-induced cell migration was determined by Transwell assay. The results showed that the migration ability of RAW 264.7 cells was markedly increased following LPS stimulation, whereas Tan IIA treatment attenuated the effect of LPS on RAW 264.7 cell migration.

Subsequently, NF- $\kappa \mathrm{B}$ mRNA and protein levels were tested in RAW 264.7 cells. The results suggested that NF- $\kappa$ B expression was upregulated following LPS stimulation and Tan IIA treatment suppressed NF- $\kappa \mathrm{B}$ activation (Fig. 5A and B). In addition, the levels of MMP-9 in RAW 264.7 cells were detected by RT-qPCR and gelatin zymography. The data indicated that the levels of MMP-9 were notably induced by LPS, while Tan IIA treatment abolished the effect of LPS on the upregulation of 

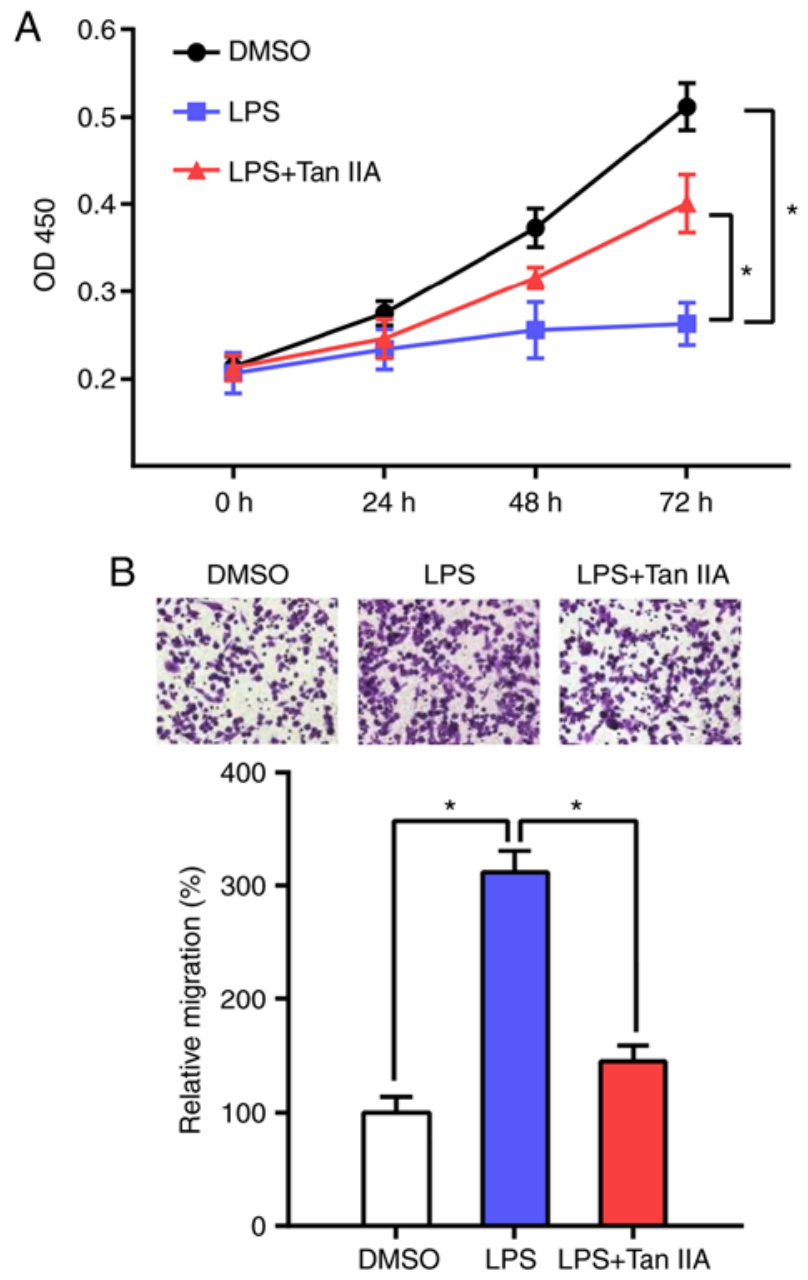

Figure 4. Tan IIA restores the proliferation and migration capacity of LPS-stimulated RAW 264.7 cells. (A) Proliferation rates of DMSO, LPS or LPS+Tan IIA-treated RAW 264.7 cells were determined by CCK-8 assay. Optical density (OD) 450 values at the indicated time points are presented as the mean \pm standard deviation from six independent experiments. "P<0.05 vs. the indicated control. (B) Cell migration capacities of DMSO, LPS or LPS+Tan IIA-treated RAW 264.7 cells were assessed by Transwell assay. Representative images are shown at the top of the histogram. Four random microscopic fields (magnification, x100) were used for cell counting (bottom histogram). Data are presented as the mean \pm standard deviation from three independent experiments. " $\mathrm{P}<0.05$ vs. the indicated control. Tan IIA, tanshinone IIA; LPS, lipopolysaccharide.

MMP-9 (Fig. 5C-E). These results suggested that Tan IIA can suppress the NF- $\kappa$ B signaling pathway elicited by LPS in RAW 264.7 cells.

\section{Discussion}

This is the first study to explore the function of Tan IIA in CAs in rat models. In the present study, it was demonstrated that Tan IIA treatment could inhibit CA formation in rats through the suppression of macrophage infiltration. In addition, Tan IIA was shown to exert an anti-inflammatory effect on CAs, manifested by the suppression of NF- $\kappa$ B expression and subsequent inflammatory activation in aneurysmal walls.

In rat models, increased hemodynamic stress at the bifurcation sites of intracranial arteries was found to cause prolonged inflammation and trigger formation and progression of CAs, which is consistent with the disease pathogenesis in humans $(17,22)$. This model is widely used to explore the mechanisms underlying human CA formation and growth. In the past few decades, numerous studies have identified the presence of inflammatory responses in CA lesions and have suggested that inflammatory processes play a vital role in the pathogenesis of CAs (5-9). NF- $\mathrm{BB}$ is a key transcription factor regulating the expression of a series of inflammation-related genes. Chronic inflammatory responses such as macrophage infiltration, cytokine release and matrix metalloproteinase expression caused by abnormally activated NF- $\kappa \mathrm{B}$ promotes $\mathrm{CA}$ formation and growth $(12,23-25)$. It has been reported that $\mathrm{NF}-\kappa \mathrm{B}$ p50 subunit deficiency or inhibition of NF- $\kappa \mathrm{B}$ activity with chimeric decoy oligonucleotides may suppress both CA formation and progression (12), which indicates that the $\mathrm{NF}-\kappa \mathrm{B}$ signaling pathway is a promising target for the clinical treatment of CA.

Tan IIA is a diterpenoid naphthoquinone found in the traditional Chinese medicine Danshen. It has diverse pharmacological effects, including anti-inflammatory, antioxidant and antibacterial, and is extensively used in China for the treatment of cancer, liver and cardiovascular diseases and diabetes (13-15). Although several studies have investigated the function of Tan IIA in different animal models of human diseases $(26,27)$, the effect of Tan IIA on CA formation and progression has not been reported. In the present study, it was found that Tan IIA treatment prevented the process of macrophage infiltration and degeneration of aneurysmal walls. This effect was due to the inhibition of NF- $\kappa \mathrm{B}$ and MCP-1 expression. Similar findings were reported in a myocardial infarction rat model (28). In addition, MMP-2 and MMP -9 expressions were also decreased in the Tan IIA-treated group. These MMPs are mainly secreted by infiltrated macrophages and play a crucial role in tissue remodeling (29). In human CAs, MMP-2 and MMP-9 expression was found to be markedly elevated in the serum and aneurysmal walls of patients (30). However, several studies have demonstrated that MMP-2 does not contribute to CA formation $(31,32)$. Although there are some conflicting studies concerning the function of MMPs in CA pathogenesis, the results suggest that Tan II treatment reduces MMP-2 and MMP-9 levels and subsequently alleviates destruction in aneurysmal walls.

Macrophages derived from blood monocytes play a vital role in all stages of the inflammatory response (33). The LPS-stimulated RAW 264.7 murine macrophage cell model is a common experimental macrophage model for anti-inflammatory drug screening. LPS can induce NF- $\mathrm{NB}$ activation and cytokine production in RAW 264.7 cells. The present data demonstrated that Tan IIA treatment reduced cytokine levels and NF- $\kappa \mathrm{B}$ expression, indicating that Tan IIA has an anti-inflammatory effect in RAW 264.7 cells. In addition, Tan IIA could reverse LPS-induced RAW 264.7 cell differentiation, which was characterized by decreased proliferation and increased migration. The expression of MMP-9 was also affected by Tan IIA. These results are consistent with the previous findings as discussed above.

In conclusion, the present findings suggest that Tan IIA attenuates inflammatory responses in CA formation through inhibition of $\mathrm{NF}-\kappa \mathrm{B}$ signaling and macrophage infiltration. These findings may furnish novel therapeutic candidates for the treatment of CAs. 

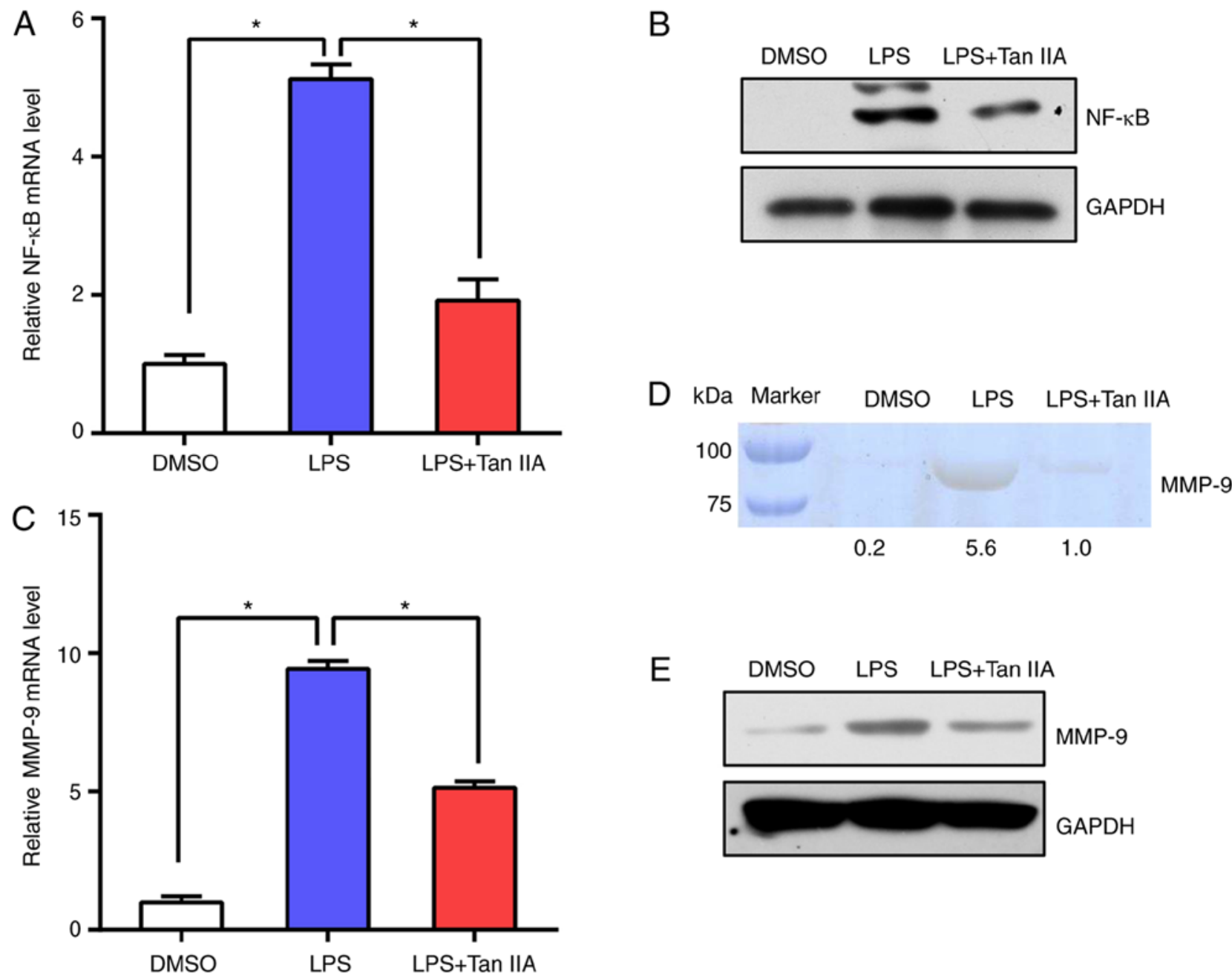

E

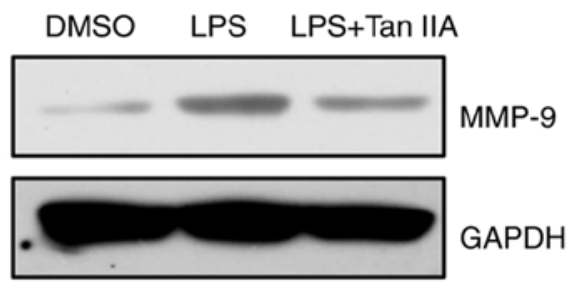

Figure 5. Tan IIA reduces NF- $\kappa$ B and MMP-9 expression in LPS-stimulated RAW 264.7 cells. (A) RT-qPCR analysis of mRNA expression levels of NF- $\kappa$ B in DMSO, LPS or LPS+Tan IIA-treated RAW 264.7 cells. Results are presented as the mean \pm standard deviation. * $\mathrm{P}<0.05$ vs. the indicated control. (B) Western blot analysis of NF- $\kappa$ B protein levels in DMSO, LPS or LPS+Tan IIA-treated RAW 264.7 cells. (C) RT-qPCR analysis of mRNA expression levels of MMP-9 in DMSO, LPS or LPS+Tan IIA-treated RAW 264.7 cells. Results are presented as the mean \pm standard deviation. ${ }^{*} \mathrm{P}<0.05$ vs. the indicated control. (D) MMP-9 activity from each cell group was measured by gelatin zymography assay. Relative gray values of each band were calculated and are shown below the blot. (E) Western blot analysis of MMP-9 protein levels in DMSO, LPS or LPS+Tan IIA-treated RAW 264.7 cells. Tan IIA, tanshinone IIA; LPS, lipopolysaccharide; NF- $\kappa \mathrm{B}$, nuclear factor- $\kappa \mathrm{B}$; MMP, matrix metalloproteinase; RT-qPCR, reverse transcription quantitative polymerase chain reaction.

\section{Acknowledgements}

Not applicable.

\section{Funding}

This study was supported by grants from the Jiangsu Province Traditional Chinese Medicine Bureau (grant no. YB2017093) and the Science and Technology Development Key Projects of Nanjing Medical University (grant no. 2016NJMUZD026).

\section{Availability of data and materials}

All data generated or analyzed during this study are included in this published article.

\section{Authors' contributions}

JM, DH, GQ and NL designed and organized the study. JM, $\mathrm{DH}, \mathrm{ZW}, \mathrm{JZ}$ and HL performed the experiments. JM, DH, ZL,
XW and YL analyzed and interpreted the data. GQ, ZL, XW and YL helped to draft the introduction and results section of the paper. JM and NL wrote and revised the manuscript. All authors read and approved the final manuscript.

\section{Ethics approval and consent to participate}

All animal experiments were performed in accordance with the Guide for the Care and Use of Laboratory Animals by the National Institutes of Health. The handling procedures were approved by the Institutional Review Board of Nanjing Medical University

\section{Patient consent for publication}

Not applicable.

\section{Competing interests}

The authors declare that they have no competing interests. 


\section{References}

1. Vlak MHM, Algra A, Brandenburg R and Rinkel GJ: Prevalence of unruptured intracranial aneurysms, with emphasis on sex, age, comorbidity, country, and time period: A systematic review and meta-analysis. Lancet Neurol 10: 626-636, 2011.

2. Baron EP: Headache, cerebral aneurysms, and the use of triptans and ergot derivatives. Headache 55: 739-747, 2015.

3. Bowles E: Cerebral aneurysm and aneurysmal subarachnoid haemorrhage. Nurs Stand 28: 52-59, 2014.

4. van Gijn J, Kerr RS and Rinkel GJ: Subarachnoid haemorrhage. Lancet 369: 306-318, 2007.

5. Pera J, Korostynski M, Krzyszkowski T, Czopek J, Slowik A, Dziedzic T, Piechota M, Stachura K, Moskala M, Przewlocki R and Szczudlik A: Gene expression profiles in human ruptured and unruptured intracranial aneurysms: What is the role of inflammation? Stroke 41: 224-231, 2010.

6. Hosaka $\mathrm{K}$ and Hoh BL: Inflammation and cerebral aneurysms. Transl Stroke Res 5: 190-198, 2014.

7. Tuttolomondo A, Di Sciacca R, Di Raimondo D, Pedone C, La Placa S, Pinto A and Licata G: Effects of clinical and laboratory variables and of pretreatment with cardiovascular drugs in acute ischaemic stroke: A retrospective chart review from the GIFA study. Int J Cardiol 151: 318-322, 2011

8. Di Raimondo D, Tuttolomondo A, Buttà C, Miceli S, Licata G and Pinto A: Effects of ACE-inhibitors and angiotensin receptor blockers on inflammation. Curr Pharm Des 18: 4385-4413, 2012.

9. Licata G, Tuttolomondo A, Corrao S, Di Raimondo D, Fernandez P, Caruso C, Avellone $\mathrm{G}$ and Pinto A: Immunoinflammatory activation during the acute phase of lacunar and non-lacunar ischemic stroke: Association with time of onset and diabetic state. Int J Immunopathol Pharmacol 19: 639-646, 2006.

10. Aoki T, Kataoka H, Shimamura M, Nakagami H, Wakayama K, Moriwaki T, Ishibashi R, Nozaki K, Morishita R and Hashimoto N: NF-kappaB is a key mediator of cerebral aneurysm formation. Circulation 116: 2830-2840, 2007.

11. Moriwaki T, Takagi Y, Sadamasa N, Aoki T, Nozaki K and Hashimoto N: Impaired progression of cerebral aneurysms in interleukin-1 $\beta$-deficient mice. Stroke 37: 900-905, 2006.

12. Aoki T, Kataoka H, Morimoto M, Nozaki K and Hashimoto N: Macrophage-derived matrix metalloproteinase- 2 and -9 promote the progression of cerebral aneurysms in rats. Stroke 38: 162-169, 2007.

13. Zhang M, Qian Y and Tang A: Research progress of pharmacologic actions of TanshinoneIIA. Med Recapitulate 16: 2661-2664, 2010.

14. Wang X: Progress of pharmacological research and clinical application of Tanshinone II A. Guang Ming J Chin Med 26: 1514-1517, 2011

15. Hosaka K, Downes DP, Nowicki KW and Hoh BL: Modified murine intracranial aneurysm model: Aneurysm formation and rupture by elastase and hypertension. J Neurointerv Surg 6: 474-479, 2014.

16. Toth M, Sohail A and Fridman R: Assessment of gelatinases (MMP-2 and MMP-9) by gelatin zymography. Methods Mol Biol 878: 121-135, 2012

17. Li Y, Gu B, Liu J, Xiong X, Zhou C and Wu G: Research progress of Tanshinone II A. Li ShiZhen Med Materia Med Res 21: $1770-1772,2010$.

18. Livak KJ and Schmittgen TD: Analysis of relative gene expression data using real-time quantitative PCR and the 2(-Delta Delta C(T)) method. Methods 25: 402-408, 2001.

19. Jin UH, Suh SJ, Chang HW, Son JK, Lee SH, Son KH, Chang YC and Kim CH: Tanshinone IIA from Salvia miltiorrhiza BUNGE inhibits human aortic smooth muscle cell migration and MMP-9 activity through AKT signaling pathway. J Cell Biochem 104: $15-26,2008$.
20. Hartley JW, Evans LH, Green KY, Naghashfar Z, Macias AR, Zerfas PM and Ward JM: Expression of infectious murine leukemia viruses by RAW264.7 cells, a potential complication for studies with a widely used mouse macrophage cell line. Retrovirology 5: 1, 2008.

21. Saxena RK, Vallyathan V and Lewis DM: Evidence for lipopolysaccharide-induced differentiation of RAW264.7 murine macrophage cell line into dendritic like cells. J Biosci 28: 129-134, 2003.

22. Jou LD, Lee DH, Morsi H and Mawad ME: Wall shear stress on ruptured and unruptured intracranial aneurysms at the internal carotid artery. AJNR Am J Neuroradiol 29: 1761-1767, 2008.

23. Chalouhi N, Ali MS, Jabbour PM, Tjoumakaris SI, Gonzalez LF, Rosenwasser RH, Koch WJ and Dumont AS: Biology of intracranial aneurysms: Role of inflammation. J Cereb Blood Flow Metab 32: 1659-1676, 2012.

24. Starke RM, Raper DM, Ding D, Chalouhi N, Owens GK, Hasan DM, Medel R and Dumont AS: Tumor necrosis factor- $\alpha$ modulates cerebral aneurysm formation and rupture. Transl Stroke Res 5: 269-277, 2014.

25. Starke RM, Chalouhi N, Ali MS, Jabbour PM, Tjoumakaris SI, Gonzalez LF, Rosenwasser RH, Koch WJ and Dumont AS: The role of oxidative stress in cerebral aneurysm formation and rupture. Curr Neurovasc Res 10: 247-255, 2013.

26. Tang FT, Cao Y, Wang TQ, Wang LJ, Guo J, Zhou XS, Xu SW, Liu WH, Liu PQ and Huang HQ: Tanshinone IIA attenuates atherosclerosis in ApoE(-/-) mice through down-regulation of scavenger receptor expression. Eur J Pharmacol 650: 275-284, 2011.

27. Ji X, Tan BK, Zhu YC, Linz W and Zhu YZ: Comparison of cardioprotective effects using ramipril and DanShen for the treatment of acute myocardial infarction in rats. Life Sci 73: 1413-1426, 2003.

28. Ren $\mathrm{ZH}$, Tong $\mathrm{YH}, \mathrm{Xu} \mathrm{W}, \mathrm{Ma} \mathrm{J}$ and Chen Y: Tanshinone II A attenuates inflammatory responses of rats with myocardial infarction by reducing MCP-1 expression. Phytomedicine 17: 212-218, 2010.

29. Ota R, Kurihara C, Tsou TL, Young WL, Yeghiazarians Y, Chang M, Mobashery S, Sakamoto A and Hashimoto T: Roles of matrix metalloproteinases in flow-induced outward vascular remodeling. J Cereb Blood Flow Metab 29: 1547-1558, 2009.

30. Jin D, Sheng J, Yang X and Gao B: Matrix metalloproteinases and tissue inhibitors of metalloproteinases expression in human cerebral ruptured and unruptured aneurysm. Surg Neurol 68 (Suppl): S11-S16, 2007.

31. Nuki Y, Matsumoto MM, Tsang E, Young WL, van Rooijen N, Kurihara $\mathrm{C}$ and Hashimoto T: Roles of macrophages in flow-induced outward vascular remodeling. J Cereb Blood Flow Metab 29: 495-503, 2009.

32. Pannu H, Kim DH, Guo D, King TM, Van Ginhoven G, Chin T, Chang K, Qi Y, Shete S and Milewicz DM: The role of MMP-2 and MMP-9 polymorphisms in sporadic intracranial aneurysms. J Neurosurg 105: 418-423, 2006.

33. Fujiwara N and Kobayashi K: Macrophages in inflammation. Curr Drug Targets Inflamm Allergy 4: 281-286, 2005.

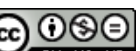

This work is licensed under a Creative Commons Attribution-NonCommercial-NoDerivatives 4.0 International (CC BY-NC-ND 4.0) License. 